\title{
Annealing of isolated amorphous zones in silicon
}

\author{
S. E. Donnelly ${ }^{\text {a) }}$ \\ Joule Physics Laboratory, Institute for Materials Research, University of Salford, Manchester M5 4WT, \\ United Kingdom \\ R. C. Birtcher ${ }^{\text {b) }}$ \\ Materials Science Division, Argonne National Laboratory, Argonne, Illinois 60439 \\ V. M. Vishnyakov and G. Carter \\ Joule Physics Laboratory, Institute for Materials Research, University of Salford, Manchester M5 4WT, \\ United Kingdom
}

(Received 4 October 2002; accepted 24 January 2003)

\begin{abstract}
In situ transmission electron microscopy has been used to observe the production and annealing of individual amorphous zones in silicon resulting from impacts of $200-\mathrm{keV} \mathrm{Xe}$ ions at room temperature. As has been observed previously, the total amorphous volume fraction decreases over a temperature range from room temperature to approximately $500{ }^{\circ} \mathrm{C}$. When individual amorphous zones were monitored, however, there appeared to be no correlation of the annealing temperature with initial size: zones with similar starting sizes disappeared (crystallized) at temperatures anywhere from $70^{\circ} \mathrm{C}$ to more than $400{ }^{\circ} \mathrm{C}$. Frame-by-frame analysis of video recordings revealed that the recovery of individual zones is a two-step process that occurred in a stepwise manner with changes taking place over seconds, separated by longer periods of stability. (C) 2003 American Institute of Physics. [DOI: 10.1063/1.1562336]
\end{abstract}

Ion implantation is a major technology for semiconductor device manufacture, but it unavoidably leads to radiation damage that must be minimized, controlled, or removed. Heterogeneous nucleation of the amorphous phase may result when an ion impact causes a sufficiently high localdefect density to render a small region amorphous. ${ }^{1-4}$ For large energy-deposition densities, this may occur for a single ion impact. As ion fluence further increases, these zones accumulate and overlap to form a continuous amorphous layer.

Thermal annealing can convert such amorphous layers to crystal by advancement of the interface with good crystal into the layer. Motion of the interface, so as to produce additional amorphization or recrystallization, can also be controlled by varying the ion flux and substrate temperature. ${ }^{5}$ This suggests that recrystallization involves the generation and movement of defects at the amorphous-crystalline $(\mathrm{a} / \mathrm{c})$ interface. Possible defects include dangling bonds, ${ }^{5}$ charged kinks on terraces, ${ }^{6}$ the so-called "bond defect," 7 and divacancies. $^{2}$

Recrystallization of incompletely amorphized layers has been observed to occur thermally and by electron irradiation. ${ }^{8}$ The annealing behavior has been studied by Rutherford backscattering, ${ }^{9}$ which determines the fractional lattice disorder, and by transmission electron microscopy (TEM) ${ }^{1}$ in which amorphous zones were observed to recrystallize at lower temperatures than the continuous amorphous layer, although details of the dynamics of this zone annealing were not recorded. The zones were also observed to recrystallize over a wide range of temperature.

The thermal recrystallization rate of the planar a/c interface exhibits Arrhenius-type behavior with a single activation energy of $2.7 \mathrm{eV}$ for recrystallization rates varying over

\footnotetext{
${ }^{a)}$ Electronic mail: s.e.donnelly@salford.ac.uk

${ }^{b)}$ Electronic mail: birtcher@anl.gov
}

ten orders of magnitude and temperatures from 500 to 1400 K. ${ }^{5}$ However, recent molecular dynamics (MD) simulations yielded two ranges of activation energies at low and high temperatures, respectively. ${ }^{10,11}$ The difference between these results was attributed to a higher concentration of defects near the interface in the MD simulation. Similarly, MD simulations of the recrystallization of small amorphous zones yielded a range of activation energies that increase with zone size. ${ }^{12}$ In these latter simulations, annealing was observed to result in periods during which no crystallization occurred, separated by periods of rapid partial recrystallization. It should be noted that MD simulations of annealing are carried out over very short time scales $(<100 \mathrm{ps})$.

The present work was undertaken to examine the kinetics of thermal annealing of isolated amorphous zones in $\mathrm{Si}$ using a TEM in which in situ ion irradiation can be carried out. Using a heating stage, the entire "life-cycle" of individual amorphous zones can be followed, from creation to recrystallization, and recorded on film and videotape.

Specimens were prepared by diamond core drilling 3-mm disks from single-side-polished, 400- $\mu \mathrm{m}$-thick, $p$-type (boron, 80 to $160 \Omega \mathrm{cm}$ ) $\{110\}$ Czochralski silicon wafers. Disks were mechanically ground to a thickness of $200 \mu \mathrm{m}$ before being dimpled and electrochemically jet-thinned to perforation in a non-acid etch. In situ ion irradiations and anneals (using a Gatan double-tilt heating stage) were carried out in a Hitachi H-9000 TEM, operating at $100 \mathrm{keV}$, located at the IVEM/Accelerator Facility at Argonne National Laboratory. ${ }^{13}$ The ion beam is oriented $30^{\circ}$ from the microscope axis. The electron energy was chosen so as to minimize electron-beam annealing of amorphous zones in silicon. ${ }^{8}$ Under our viewing conditions, no effects of the electron beam on the amorphous zones were observed over a time scale of $30 \mathrm{~min}$. During isochronal annealing experi- 


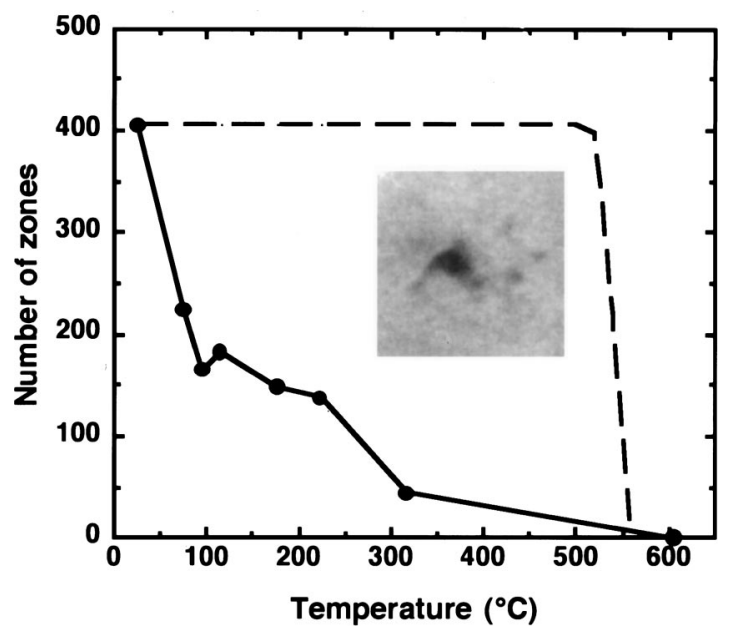

FIG. 1. Number of amorphous zones surviving 10-min anneals to successively higher temperatures. The dashed line is calculated behavior for a singly activated process with activation energy of $2.7 \mathrm{eV}$. Inset shows contrast resulting from the impact of a single $200-\mathrm{keV}$ Xe ion (box width $=30 \mathrm{~nm}$ ). Image was recorded under bright-field, down-zone conditions $(\langle 110\rangle$ zone).

ments, the electron beam irradiated the specimens only for the short periods necessary to realign the specimen and record images on film. Amorphous zones were imaged using structure-factor contrast ${ }^{14}$ under down-zone, bright-field conditions, ${ }^{15}$ with the electron beam normal to the $\{110\}$ surface. Images were recorded either on photographic film or on S-VHS videotape by means of a Gatan 622 camera and image intensification system with a frame rate of 30/s. The recrystallization behavior of a large number of individual zones was followed on a series of negatives following different isochronal annealing steps. Recrystallization of a smaller number of zones was followed in real time during a slow temperature increase by recording on videotape.

The projected area of each zone was determined by tracing a boundary around individual zones by hand (using Adobe Photoshop ${ }^{\mathrm{TM}}$ on an Apple Macintosh PC). The areas contained within individual boundaries were determined using the software NIH IMAGE. There is likely to be a slight systematic underestimation of the actual size of zones because a minimum thickness of amorphous material is required to yield a discernable contrast.

The inset micrograph in Fig. 1 shows an image, digitized from video, of the contrast resulting from the impact of a single $200-\mathrm{keV} \mathrm{Xe}$ ion. The Monte-Carlo simulation program SRIM ${ }^{16}$ indicates that collision cascades resulting from a 200-keV Xe irradiation will have a subcascade structure, and this is reflected in the observed contrast, which consists of a large area and a number of smaller ones. As the number of such patches of contrast increases, a concomitant amorphous ring is observed in the diffraction pattern, confirming the identification of contrast as amorphous pockets in crystalline silicon.

Isochronal anneals were carried out on a specimen irradiated to a fluence of $1.25 \times 10^{12}$ ions $\mathrm{cm}^{-2}$. For each temperature step, the specimen was heated to the target temperature as rapidly as possible (1-2 $\mathrm{min})$, and the temperature was maintained for $10 \mathrm{~min}$. The specimen was then cooled to below $40^{\circ} \mathrm{C}$ and realigned for down-zone, structure-factor imaging. The projected areas of more than 400 zones were Downloaded 06 Jun 2003 to 146.87.123.133. Redistribution subje

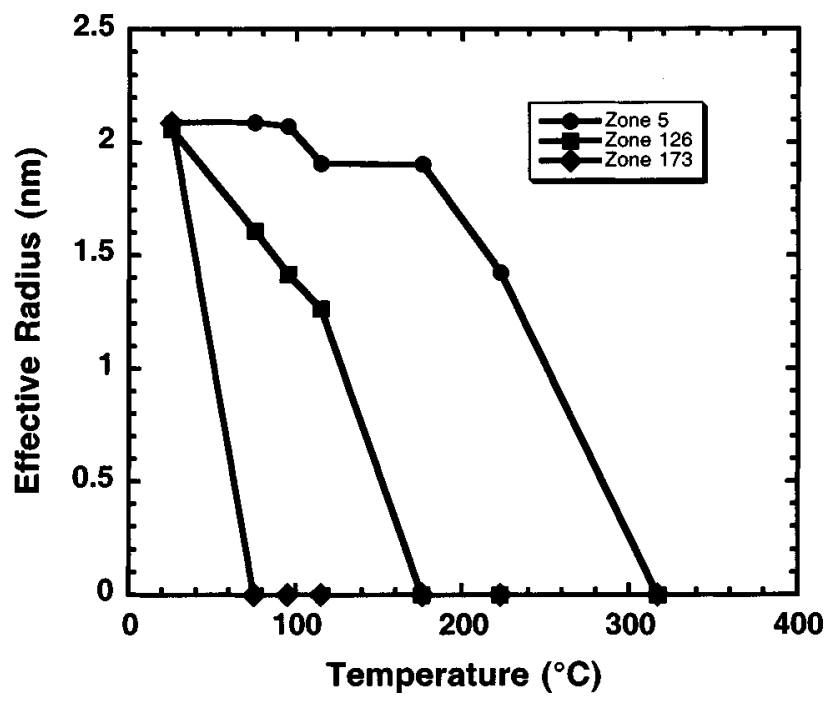

FIG. 2. Change in effective radius of three individual amorphous zones with similar starting radius following 10-min anneals to successively higher temperatures.

measured and converted to effective radii by assuming a spherical shape. The number of amorphous zones remaining following each annealing step is illustrated in Fig. 1. This behavior is in good agreement with results obtained previously. ${ }^{1}$ Also plotted in Fig. 1 is a dashed line indicating the theoretical annealing behavior of these zones if the motion of the a/c boundary followed the same kinetics as in planar geometry.

Two important points are observable on this plot: first, an activation energy of $2.7 \mathrm{eV}$ would result in all zones surviving to above $500^{\circ} \mathrm{C}$, and second, the temperature range over which the zones would recrystallize due to a singly activated process would be much smaller than is actually observed. Clearly, the recrystallization behavior of small amorphous zones is very different from that of the planar a/c interface and does not involve a single, well-defined activation energy.

The averaged information shown in Fig. 1 fails to reveal the erratic annealing of individual zones. This is illustrated in Fig. 2, where the annealing behavior of three amorphous zones of almost identical starting sizes (effective radius of just over $2 \mathrm{~nm}$ ) is plotted. This wide range of recrystallization behavior is typical. Analysis of more than 400 zones on each annealing step reveals little consistency in their behavior and little or no correlation between their size and recrystallization temperature. On each annealing step, zones of every size disappear. The behavior is thus neither consistent with a singly activated process nor with the findings of MD simulations in which the activation energy for recrystallization was seen to increase with zone size. ${ }^{12}$

The dynamical shrinkage of individual zones was monitored by slowly ramping the temperature while recording the image on videotape. Figure 3 shows the behavior of a single isolated amorphous zone (initial effective radius $1.7 \mathrm{~nm}$ ) as a function of time during a brief period of just over a minute, during which the temperature rose from 302 to $308^{\circ} \mathrm{C}$. Shrinkage of this zone took place in two distinct stages labeled "A" and "B." Each stage lasts on the order of $1 \mathrm{~s}$, and the zone changed little during the period between the two stages. In general, during a slow temperature ramp, zones to AIP license or copyright, see http://ojps.aip.org/aplo/aplcr.jsp 


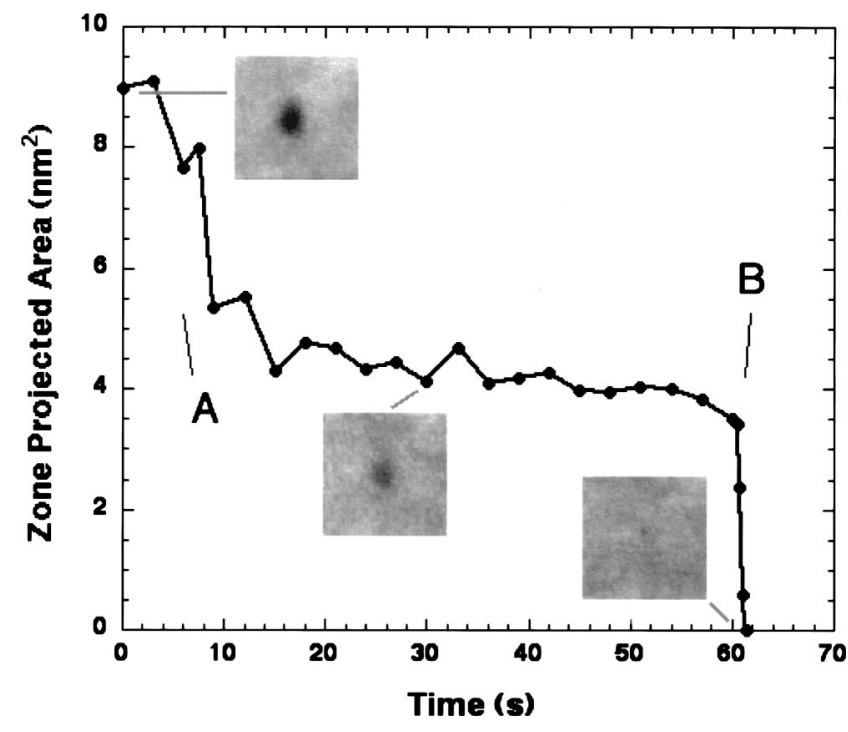

FIG. 3. Recrystallization behavior of a single isolated amorphous zone (initial effective radius $1.7 \mathrm{~nm}$ ) as a function of time during a period of just over a minute, during which the temperature rose from 302 to $308^{\circ} \mathrm{C}$.

shrink during short periods $(\approx 1 \mathrm{~s})$ and then remain unchanged for much longer periods $(\approx$ minutes). This type of behavior, albeit for smaller zones, at lower temperatures, and over much smaller time scales (tens of picoseconds), was found in MD simulations of the annealing of amorphous zones in silicon. ${ }^{12}$ The present work represents an experimental observation of this type of sporadic recrystallization.

Recrystallization involves a triggering event, followed by a rapid motion of the $\mathrm{a} / \mathrm{c}$ interface. The triggering event may be the creation of a defect whose subsequent migration at the interface results in some recrystallization. In the case of the planar a/c interface, both the creation and migration energies of the defect responsible for recrystallization are apparently well defined, and their sum yields an activation energy of $2.7 \mathrm{eV}$. However, the MD calculations ${ }^{10,11}$ indicate that when there is a high concentration of defects present near the a/c interface, recrystallization does not have a welldefined activation energy. When a single heavy-ion impact results in an energy density sufficient for an amorphous zone to form, the surrounding region, in which the energy density is lower, will have a high density of point defects. Such a configuration is seen in MD simulations. ${ }^{17}$ The precise atomic arrangement near the interface will vary from zone to zone and from point to point around the surface of each zone. The energy required to create the trigger defect responsible for recrystallization will thus vary from zone to zone (as possibly will the migration energy).

The time for the rapid recrystallization steps (as seen in Fig. 3) is on the order of $1 \mathrm{~s}$ at $300^{\circ} \mathrm{C}$. Annealing of a nanometer-sized amorphous volume involves several hundred atomic rearrangements. At a temperature of $300^{\circ} \mathrm{C}$, a singly activated process with a rate constant of $10^{13} \mathrm{~s}^{-1}$ that requires between 100 and 1000 atomic jumps to go to completion, would have an activation energy in the range 1.14 to $1.25 \mathrm{eV}$. A migration energy of $1.2 \mathrm{eV}$ has been measured for the defect responsible for both thermal and ion-beam-induced recrystallization (implying a formation energy of $1.5 \mathrm{eV}$ ). Although Jackson suggests that this defect is the dangling bond, ${ }^{5}$ it should be noted that this is generally assumed to have a creation energy of $2.25 \mathrm{eV}$ (half the cohesive energy), which implies a migration energy of $0.43 \mathrm{eV}$.

We have shown that recrystallization of nanometer-sized amorphous zones is not a singly activated process, but one that takes place over a wide range of temperatures. It involves at least two thermally activated processes: a triggering event and the recrystallization process itself. Triggering events occur over a temperature range of more than $300^{\circ} \mathrm{C}$. The defect responsible for recrystallization has not yet been identified, but the dangling bond, ${ }^{5}$ the kink site, ${ }^{6}$ and the bond defect ${ }^{7}$ have all been suggested as possible candidates. The irregularity of the interface of any nanometer-sized zone and the variation in local atomic arrangement at the interface may be responsible for a wide range of energies for the creation of the defect responsible for recrystallization. This creation is the rate-limiting triggering event. The subsequent rapid recrystallization appears to have an associated energy of about $1.2 \mathrm{eV}$.

We thank E. Ryan, B. Kestel, A. McCormick, and L. Funk for technical assistance. One of the authors (S. E. D.) acknowledges funding from Argonne National Laboratory for extended visits to the laboratory. This research was supported by the U.S. Department of Energy, Office of Science, BES-Materials Science, under Contract No. W-31-109-Eng38.

${ }^{1}$ L. T. Chadderton, Radiat. Eff. 8, 77 (1971).

${ }^{2}$ O. W. Holland, S. J. Pennycook, and G. L. Albert, Appl. Phys. Lett. 55, 2503 (1989)

${ }^{3}$ F. F. Morehead and B. L. Crowder, Radiat. Eff. 6, 27 (1970).

${ }^{4}$ L. M. Howe and M. H. Rainville, Nucl. Instrum. Methods 182/183, 143 (1981).

${ }^{5}$ K. A. Jackson, J. Mater. Res. 3, 1218 (1986).

${ }^{6}$ J. S. Williams, in Surface Modification and Alloying, edited by J. M. Poate and G. Foti (Plenum, New York, 1984), pp. 133-163.

${ }^{7}$ B. Weber, D. M. Stock, and K. Gärtner, Mater. Sci. Eng., B 71, 213 (2000).

${ }^{8}$ I. Jencic and I. M. Robertson, J. Mater. Res. 11, 2152 (1996).

${ }^{9}$ F. Priolo, A. Battaglia, R. Nicotra, and E. Rimini, Appl. Phys. Lett. 57, 768 (1990).

${ }^{10}$ N. Bernstein, Ph.D. thesis, Harvard University, Cambridge, Massachusetts, 1998

${ }^{11}$ N. Bernstein, M. J. Azziz, and E. Kaxiras, Phys. Rev. B 61, 6696 (2000).

${ }^{12}$ M. J. Caturla, T. Diaz de la Rubia, L. A. Marqués, and G. H. Gilmer, Phys. Rev. B 54, 16683 (1996).

${ }^{13}$ C. W. Allen, L. L. Funk, E. A. Ryan, and S. T. Ockers, Nucl. Instrum. Methods Phys. Res. B 40/41, 553 (1989).

${ }^{14}$ M. F. Ashby and L. M. Brown, Philos. Mag. 8, 1083 (1963).

${ }^{15}$ M. W. Bench, D. K. Tappin, and I. M. Robertson, Philos. Mag. Lett. 66, 39 (1992)

${ }^{16}$ J. F. Ziegler, J. P. Biersack, and U. Littmark, The Stopping and Ranges of Ions in Solids (Pergamon, Oxford, 1985), see [http://www.srim.org/].

${ }^{17}$ T. Diaz de la Rubia and G. H. Gilmer, Phys. Rev. Lett. 74, 2507 (1995). 